Revue de philosophie et de sciences humaines

e-Portique | 2005

\title{
La déréliction du langage dans le penser politique en Afrique
}

Dr Samba Diakite

\section{(2) OpenEdition}

Journals

Édition électronique

URL : https://journals.openedition.org/leportique/521

DOI : 10.4000/leportique.521

ISSN : $1777-5280$

\section{Éditeur}

Association "Les Amis du Portique"

Référence électronique

Dr Samba Diakite, "La déréliction du langage dans le penser politique en Afrique », Le Portique [En ligne], e-Portique, mis en ligne le 12 mai 2005, consulté le 10 septembre 2022. URL : http:// journals.openedition.org/leportique/521 ; DOI : https://doi.org/10.4000/leportique.521

Ce document a été généré automatiquement le 10 septembre 2022

Tous droits réservés 


\title{
La déréliction du langage dans le penser politique en Afrique
}

\author{
Dr Samba Diakite
}

1 Mots clés

2 Afrique-déréliction-langage-culture-crises-politique-démocratie-liberté-concordeguerres- engagement -promesse-ruse-raison-éthique- penser - domination - Droit respect- intégration.

Introduction

3 Au cœur de la philosophie, déjà si confrontée à sa propre définition, la philosophie politique apparaît comme le comble de l'ambiguïté, de l'indécision et du déchirement du langage. Depuis l'Antiquité, PLATON posait la difficile problématique de l'éthique langagière :comment des discours faux (des sophistes), s'interrogeait-il, pouvaient s'avérer plus persuasifs et plus perspicaces que des discours vrais (de SOCRATE) ? On sait aussi comment ce dernier a été condamné à boire la ciguë par "la polymathie" apparente des sophistes. C'est dire que le langage, s'il est paradoxologique, est avant tout discriminant; et comme tel, il demeure en effet, le premier problème de la philosophie politique et de la Philosophie tout court.

4 La politique ne peut se passer du langage. D'ailleurs, la démocratie, tant vénérée par les sociétés modernes ne peut véritablement s'exercer sans la distribution de la parole, sans la diversité des opinions. Le paradoxe du langage se résume ainsi en ceci :sans le langage , rien n'est possible , il n'y a pas de pensée politique ; avec le langage et par le langage, tout est possible même le pire. Ainsi la condition humaine est -elle déterminée aussi bien par notre dire que par notre écrit, si nous définissons brièvement le langage selon le Robert, comme « fonction d'expression de la pensée et de communication entre les hommes, mise en œuvre par les organes de la phonation(parole) ou par une notation au moyen des signes matériels (écriture) ». Devant cette forêt de possibilités $\mathrm{du}$ langage où toutes les déviations paraissent justifiables, où la conquête du pouvoir semble se fonder sur le camouflage, les propagandes, le caprice et sur l'isothénie des arguments, comment la philosophie en tant que ré-flexion peut-elle penser le dire politique en Afrique? Si le langage politique peut paraître comme un abîme 
indifférencié, comment lutter contre la dégénérescence du penser politique dans un système ou un régime chaotisant? A la vérité, le langage est rupture, il est facteur de guerre ou de paix, parce qu'il est essentiellement le point critique de la pensée.

L'homme a toujours parlé mais il y 'a des moments où le dire est beaucoup pris au sérieux. Ce moment, c'est lorsque le penser devient Ėthique, lorsqu'il accepte de se séparer de soi-même pour accueillir d'autres séparations dans le langage. Tel est le sens et la visée fondamentale de la démocratie, une démocratie imposée aux Ėtats africains dans les années 90. Des Ėtats africains, à qui l'Occident recommandait l'acceptation de la distribution de la parole par l'instauration des régimes pluralistes où selon Maurice DUVERGER, « la lutte politique se déroule au grand jour, librement, à ciel ouvert. On y trouve d'abord plusieurs partis politiques:d'où le nom de régimes "pluralistes » :il peut y en avoir plus de deux, mais il y en a toujours au moins deux. La lutte est publique et ouverte, également sur le plan de la presse et des moyens d'expression et d'information :les régimes pluralistes sont aussi des régimes libéraux, c'est-à-dire des régimes où existent des libertés publiques, permettant à chacun d'exprimer librement ses opinions, par la parole, l'écrit, l'adhésion à des organisations, la participation à des manifestations publiques, etc. ${ }^{1}$ Désormais, la parole est donnée, et elle peut se prendre et s'affirmer comme dé-voilement, reconstruction, manifestation des intentions et diversité des possibles. Dès lors, le langage en tant que langage de la raison, se transmue en un langage de la liberté, c'està-dire un langage de l'incohérence, un discours manipulateur, ouvert mais bien apophatique, le discours du vrai et du faux, du faux plus que du vrai dans le penser politique. On se rend ainsi compte que l'introjection de la démocratie comme fond insaisissable du discours dans la pureté de l'être se saisit dans son échec en instaurant des crises politiques aiguës en Afrique. Dans cette Afrique-là, l'unité est brisée, divisée, séparée d'elle-même, ouverte à l'Autre mais fermée en son propre fond. Autrement dit, le discours politique en Afrique est un langage déchiré, dépravé, dépourvu de l'éthique du penser car l'homme politique africain «ne fait que pousser à son paroxysme un mouvement de décomposition du langage et du mot, mouvement qu'il accélère dans une volonté de surenchère cherchant à transformer une passivité subie en un hasard prometteur délibérément déclenché. Il n'est pas exagéré de dire que nous nous trouvons aujourd'hui , pas seulement devant un langage de réduction, mais devant une déréliction du langage $» .^{2}$ Le problème est donc de savoir l'impact de la pragmatique du langage dans le jeu politique en Afrique. Le déchirement du langage n'est- il pas le fondement des crises politiques en Afrique ? Le dire dans le penser politique en Afrique n'est- il pas un jeu acrobate dangereux, une prosopolepsie? Le langage comme intransigeance du tout ou rien n'est- il pas en lui-même et en son fond, illégal et illégitime pour n'advenir que comme un encagement? Les crises politiques en Afrique ne sont-elles pas d'abord et avant tout des mal-entendus, ne sont-elles pas comme "analyseurs-révélateurs" d'un système de pourrissement du langage dans une société africaine où les acteurs semblent être des malentendants? Le malentendu politique n'a $\mathrm{t}$ - il pas pris la forme d'un langage fragmentaire, discontinu et discourtois? Ne s'est- il pas mué en des fantasmes dont le discours est formulé dans un discours hypocrite, doublé d'un ressassement vide et chaotisant? Si donc il y a des crises politiques en Afrique, c'est bien parce que notre penser politique , notre " action n'est que le " raté " du langage, car ce qui se fait est né de ce qui a déjà été dit et meurt de ce qui est en train de se dire. Il s'agit donc de réfléchir sur le sens du « dire »afin de comprendre celui $d u$ « faire » et de ne pas tomber dans les hystéries du « faire faire »ou du « faire dire »». 
${ }^{3}$ Il s'agira de réfléchir sur le problème de l'éthique du langage non pour se fourvoyer dans un dilettantisme ou un faire valoir politique mais pour dénoncer la tragédie du langage comme support de la déréliction du politique dans l'Afrique actuelle.

I/ Le Dire politique comme perversité de l'éthique du langage

6 Dans l'Afrique actuelle, avec l'avènement de la démocratie, l'éducation est en désuétude car les référentiels moraux sont en train de chuter. Nous appelons référentiels moraux, le respect de la chose donnée et de la chose jugée, de l'engagement pris, de la nécessité du dialogue pour résoudre les différends, de la préférence de la vérité au mensonge, de l'essence à l'apparence. Mais, de nos jours, la contestation devient de plus en plus récurrente. Nous ne disons pas qu'il faille avoir une société muette ;cela reviendrait à souhaiter des Ėtats autocratiques et par conséquent, l'on retournerait à la case de départ. Mais ce que nous voudrions faire remarquer est que " au nom de la politique», la contestation de la jeunesse devient une « clochardisation» de la parole, une inféodation à tel ou tel parti politique, à tel ou tel groupe tribal. Dans un tel cas, elle tend à devenir comme le dit Herbert MARCUSE, «souvent puérile et clownesque. Bien sûr, cette puérilité, cette clownerie apparaissent à l'évidence comme partie intégrante d'actes de protestation authentiques, dans des situations où l'opposition radicale se trouve isolée et outrageusement faible, en face d'un ennemi omniprésent et outrageusement fort. «La maturité » est, par définition le fait du Système, de ce qui est $»^{4}$. Ce qui est, est que la jeunesse n'a plus de repères. Les écoles et les universités, temples du savoir, sont aujourd'hui devenues les champs de bataille politique. Les arguments, les idées, n'ayant plus de substance, se diffractent, se fondent comme une glace sous le soleil ardent des tropiques et coulent bruyamment dans l'océan de la violence. ${ }^{5}$

7 En politique comme partout ailleurs, la prise de la parole est le commencement de la rupture. La question fondamentale étant de savoir qui parle; le «disant» se trouve d'emblée au centre d'un questionnement, dans le filet d'une problématique. "C'est comme si l'on jetait un filet, mais le pêcheur risque toujours d'être entraîné et de se retrouver en pleine mer quand il croyait arriver au port $» .^{6}$ Dans cet océan de l'opinion, comment atteindre la rive sans se noyer? Comment sortir de cet imparfait sans fond sans être pris au piège de son propre dire? Dans un tel cas, parler c'est s'opposer aux autres. Dès lors, tout discours est une exclusion dans la mesure où l'accès à la parole implique la déconstruction du dire et du vouloir -dire de l'autre, cet autre du dire qui va sans dire ce qu'on ne saurait dire. La parole est trahison, trahison parce que l'homme politique est hypocrite.

8 La trahison et l'hypocrisie du penser politique avec ses procédés falsificateurs, sont d'autant plus courants en Afrique que chaque homme politique se considère comme un renard tandis que le peuple , ce prétendu "phénix des hôtes » de l'Ėtat, ce « corbeau » des fables de la Fontaine, s'entre-déchire pour un pseudo-fromage qu'il ne mangera pas, probablement qu'il ne verra jamais. N'est- ce pas que «tout flatteur vit aux dépens de celui qui l'écoute ${ }^{7}$ ?

10 Le mensonge politique s'exacerbe lors des joutes électorales, le moment bien choisi pour promettre à une population majoritairement misérable, affamée et analphabète, toutes sortes de possibilités. Mais le candidat, une fois élu, la promesse devient précaire; la parole n'est plus respectée et l'engagement devient un encagement. Pourtant, la sagesse africaine, dans son fond éthique, admet que la promesse n'a de 
sens que si elle est tenue, qu'une parole n'a de valeurs que si elle est respectée et par conséquent, un homme n'a de dignité que s'il respecte sa parole "donnée ». Réalité sociale irréductible, le non respect de la promesse implique nécessairement des conséquences. Une société d'êtres inconstants et insincères ne devrait s'attendre qu'à des crises d'autorité échafaudées par la déréliction du langage.

11 Dans cette Afrique politique actuelle, le culturel et le moral, du point de vue du langage, sont une abdication du penser et semblent ne plus témoigner du respect. La politique tend à être oublieuse de l'éthique dans la mesure où le langage est a-moral. Dans un tel cas, la culture croise nécessairement la violence car on lui a enlevé sa véridicité, on a falsifié son fondement, on a travesti son essence. La culture africaine devient une Ombre, Ombre de sa propre transmutation, Ombre de soi parce que Ombre de son propre langage disqualifié. Le langage devenu Ombre est un langage obscur ,divaguant, incompréhensible parce que renversé. C'est un devenir-fantôme en attente de ses prochaines victimes. Le penser politique aurait-il besoin de meurtres pour se fonder et se valoir? N'est-ce pas là le commencement de la manifestation d'un dégoût cynique? En réalité, le non-respect de la parole donnée, l'abâtardissement du langage est , comme le dit Vladimir JANKĖLĖVITCH , «la frénésie d'une conscience dédoublée, crucifiée, déchirée par son insoluble contradiction. Plus la valeur est apparemment sacrée et révérée comme telle, plus scandaleuses et triviales sont les manifestations du dégoût cynique :cracher, vomir et rejeter! Aucun geste n'est assez énergique pour exprimer le dégoût cynique, la volonté cynique d'expulser de notre vie, de notre substance, d'éliminer de notre être en général les valeurs réputées les plus saintes :les valeurs morales sont considérées comme allant en sens contraire de la vie $»^{8}$.

12 "Le - changer -l'- Afrique "ne consiste pas à enterrer ni les engagements qu'on a pris solennellement devant le peuple, ni à consolider des soupapes de frustration pour diviser le même peuple afin de mal régner. L'émancipation du discours n'est pas ce qu'il est convenu d'appeler aujourd'hui en Afrique, «la caméléonisation» du penser politique, après « la léopardisation », concepts célèbres qui expliquent l'inconstance, le mimétisme et la prédation de l'homme-politique africain. En effet, du caméléon, nous savons ceci : c'est un terme qui émane du mot grec "khamaileôn" dont le sens exact est le «lion qui se traîne à terre ", le mot "khamai » voulant dire "à terre ». Le caméléon est donc, selon le Dictionnaire Robert, un reptile saurien qui a la faculté naturelle de changer de couleur. Il se distingue de son allure majestueuse, de sa patience à atteindre sa destination mais aussi de son regard malicieux, argile et omniprésent qui lui permet de voir tout ce qui l'entoure afin de pouvoir se confondre avec son milieu par le jeu infini du mimétisme. De manière apparente, il est inoffensif, voilà pourquoi pour les grecs, il traîne à terre. Mais, il demeure un lion parce qu'il est le roi du camouflage, de la ruse par le jeu du mimétisme.

13 En effet, le camouflage est une forme de combat qui consiste à défendre son point de vue contre les autres de manière malicieuse. Maurice DUVERGER nous fait savoir que c'est une technique qui a pour méthode de dissimuler des intérêts particuliers, des buts et des motifs d'une minorité sous de faux prétextes, de pseudo-motifs, un objectif politique derrière un autre objectif politique, plus noble et plus avouable que le premier qui tienne compte de l'adhésion populaire. "Très souvent, nous dit-il, ce procédé de camouflage prend la forme de «l'épouvantail ». On invente « un ennemi », on grossit l'importance d'un ennemi réel, en justifiant par la nécessité de se défendre contre lui des mesures prises en réalité dans l'intérêt des classes au pouvoir. En criant 
au «loup », on détourne l'attention du voyageur, et on peut lui dérober ses bagages pendant qu'il songe seulement à se préserver de l'animal. ${ }^{9}$

$14 \mathrm{Du}$ léopard, nous notons ceci: le mot vient du latin «leopardus, de leo » qui signifie «lion» et « pardus », qui veut dire "panthère ».Le léopard est donc un animal carnassier analogue au lion et qui se voit fréquemment dans les jungles africaines. Il est un prédateur très habile, prompt à affronter sa proie. Fin coureur, mais il s'essouffle vite. A l'analyse, ces deux animaux ont ceci de commun: l'agilité, la patience à atteindre son but, la Majesté, la domination par la couleur. Assimilables aux lions, selon leur étymologie, ils règnent en exaltant leur spécificité ontologique: le mimétisme et les rayures, la peur du milieu et la violence sur le milieu. De ces remarques, il faut tirer les conclusions suivantes:l'homme politique africain au pouvoir, est aussi bien caméléon que léopard. Maître du camouflage, il est insincère et inconstant parce qu'il est ce prédateur qui veut le tout ou ne laisse que le presque-rien ; Roi de la terreur, parce que, insincère et inconstant, il utilise le mensonge comme moyen de manipulation et de conservation d'un pouvoir à génuflexion, d'un pouvoir qui «fait de l'être humain tout entier- de son intelligence et de ses sens-un objet d'administration , embrayé sur la production et la reproduction non des seuls objectifs mais aussi des valeurs et des promesses du système, de son paradis idéologique. Sous le masque technologique, sous le masque politique de la démocratie, apparaît la réalité de la servitude universelle, la dissolution de la dignité humaine en une liberté de choix préconditionnée. Et la structure du pouvoir n'est plus «sublimée » dans le style d'une culture se voulant libérale, elle n'est même plus hypocrite (ne sauve même plus des « apparences » polies , l'enveloppe de la dignité), elle est brutale et ne prétend plus le moins du monde à la vérité et à la justice ». ${ }^{10}$

16 Ainsi l'abîme infini du langage qui se donne en spectacle, du dire-faux, du faux-dire, $\mathrm{du}$ penser-faux et du faux-penser conduit théâtralement à de récurrentes crises politiques dont les fondements sont les mêmes : le déchirement du langage.

II/ De la prosopolepsie aux crises politiques en Afrique

17 En Afrique, aujourd'hui, l'on tend à confondre le pouvoir moderne et l'ordre ancien. Si hier, le pouvoir était une affaire de castes, un legs voire une transmission de père en fils, les choses sont autrement aujourd'hui, surtout dans les régimes dits démocratiques. Pourtant, certains hommes politiques continuent d'imposer à toute une population, des leaders qu'elle n'a pas choisis. En effet, après la mort de JOSEPH DESIRĖ KABILA en République Démocratique du Congo, son fils est désigné par l'armée pour le remplacer sans tenir compte de l'avis d'un peuple meurtri, qui , après avoir applaudi le départ d'un pseudo-révolutionnaire a vite fini par déchanter. Mais l'une des plus graves dérélictions politiques vient d'être constatée au Togo. En effet, comme dans un régime monarchique, l'armée décide de remettre le pouvoir , après le décès du père, au fils du président EYADEMA sans vote, au mépris des lois constitutionnelles, de toute juridiction. La constitution, l'acte de naissance de l'Ėtat, le seul langage du peuple (dans les démocraties sérieuses), selon ROUSSEAU, est mise en veilleuse si elle n'est pas totalement édulcorée.

19 Ici encore, on fait et défait les lois selon la volonté du plus fort et de l'ethnie majoritaire, même si la Nature avait prévu autre chose. La domination devient héréditaire et le peuple n'a pas le droit de s'affranchir. Le règne de la terreur devient 
souverain et légal. Nombres de régimes politiques africains nous ont habitué à ces phénomènes sensationnels et inédits. Comme le dit HANNAH ARENDT , "nous sommes en présence d'un genre de régime totalement différent. Il brave, c'est vrai toutes les lois positives jusqu'à celles qu'il a lui-même promulguées.(...)Mais il n'opère jamais sans avoir la loi pour guide :car il prétend obéir rigoureusement et sans équivoque à ces lois de la Nature et de l'Histoire dont toutes les lois positives ont toujours été censées sortir ».11

20 Dans une telle situation, tout devient de l'ombre. La lutte politique devient masquée car le langage a été perverti. Dès lors, les antagonismes, au lieu de s'affaiblir, ne font que s'accentuer. L'unité se trouve brisée et il n'y a plus de soupape de légitimité et de sûreté. Nous sommes dans une situation de communication déformée où le langage va de pair avec l'a-raisonnement, où les travestissements idéologiques de la domination prennent les formes de légitimité et de légalité d'un pouvoir en treillis. La parole n'est plus en situation idéale « dans laquelle toutes les prétentions à la validité se réunissent en tant que prétentions fondamentales au raisonné. " ${ }^{12}$ Parce qu'elle est l'enjeu et le socle du pouvoir, la parole saine méconnaît la «force » d'une politique stratifiée, elle se refuse d'être la complice d'un logos »lacunaire ", le masque d'une absence de fondement.

21 Le jeu de masque du penser politique qui fait et défait les lois fondamentales selon la volonté de la "force", se donne à déchiffrer comme une superfluité, comme «ce qui est forclos, le procès d'une écriture sans origine assignable , sans tracé prescrit(...) Il ne peut jamais se lire dans le champ d'une logique de l'identité $»^{13}$. L'on se retrouve dans une fiction politique où la mise en scène devient grotesque et décadente dans la mesure où elle reste prise dans les rets de sa propre duperie. Ainsi, le jeu politique en Afrique, devient dionysiaque, un jeu théâtral sans fond, sans normes où les spectateurs se confondent aux acteurs et où la violence s'incruste pour contrecarrer l'excès dionysiaque. Le tragique tyrannique se substitue à l'espérance démocratique. Un pouvoir issu de cette sournoiserie de l'intelligence «abolit sa propre légitimité en cernant son non-lieu ;c'est-à-dire déjoue tout respect d'une origine, d'une paternité ou d'une propriété (tout ce qui tenait lieu de justification d'un fondement ).Dans cette mise à jour des instances , se dénonce également tout "vouloir-dire", toute volonté simple de sens $»^{14}$.

22 Comment supporter l'irrationalité éthique d'un langage sans mesure, d'un langage figuré parce que défigurant ? Comment un tel penser politique peut-il éviter la révolte et le ressentiment ?Si en politique, il se joue un jeu(et il s'en joue un), ne faut-il pas clairement poser les règles du jeu ?En effet, le dédoublement du langage dans le champ politique conduit l'homme politique à se donner lui-même en spectacle. La ruse de la raison se retourne contre elle-même. Le surgissement de la haine qui tient lieu de pensée dépasse l'exprimable. Le génocide rwandais, la guerre civile du Liberia et les autres rébellions en AFRIQUE ne sont-elles pas nées des frustrations et des ressentiments ?Tout se passe comme si l'Autre était inapte à vivre, comme s'il était une punaise qu'on doit exterminer par la machette ou par les fusils. On assassine les individualités, on détruit les spontanéités et « l'ennemi objectif » est la tribu de l'Autre, son ethnie ou les adeptes de sa religion . Les guerres civiles en Afrique, ont fait ces dix dernières années, plus de victimes que les camps de concentration d'Auschwitz et le Goulag. Les attaques contre la personne morale par les invectives, les diffamations et les propagandes n'ont qu'une seule visée, le meurtre de la personne juridique. Car le 
but du système est de détruire les droits civils de certaines populations en les poussant à vivre dans la désolation, dans l'isolement et d'accepter le sens de la résignation. «Leur cynisme moral, leur croyance que tout est permis, repose sur la conviction solide que tout est possible $»{ }^{15}$

23 Nous vivons dans une réalité brouillée par la monstruosité du langage où il suffit de parler pour être accusé où on ignore toute différence entre la loi et la volonté du chef. $\mathrm{N}$ 'est-ce pas que là où commence la déréliction du langage commence l'arbitraire ? Dans certains de nos États, le Droit c'est ce qui est bon pour le chef. Mais on ne peut mentir impunément au monde entier, on ne peut se jouer indéfiniment du peuple surtout si le caractère factice de la réalité rend le jeu politique superflu et où la terreur renforce les propos, où la morale est profondément enkystée et qu'on ne peut l'en extirper que par la douleur. L'illusion de l'intégration finit par faire dévoiler le visage d'un régime en masque, "un régime conjoncturel " où la vengeance fonde un retour éternel dès que survient une crise. Mais, comme le dit MARCUSE, « s'il est vrai que le peuple doit se libérer de sa servitude, il est tout aussi vrai qu'il doit d'abord se libérer de ce qu'on a fait de lui dans la société où il vit. Cette libération préalable ne peut être "spontanée", car une telle spontanéité n'exprimerait que les valeurs et les objectifs dérivés du système établi. L'autolibération est auto-éducation mais, en tant que tel, elle suppose d'abord éducation par autrui . Dans une société où l'inégal accès au savoir et à l'information fait partie de la structure sociale, la distinction et l'antagonisme entre éducateurs et éduqués sont inévitables $» .{ }^{16}$ Comment donc traduire le penser politique en Afrique en un langage neuf capable de faire passer le message de la liberté et de la dignité humaine ? Comment faire que le jeu politique- langagier soit vraiment un jeu qui ne choque plus, un jeu inoffensif ?La politique est sérieuse lorsque le langage est cordial, lorsque les engagements pris ou les accords signés sont respectés car l'affaiblissement des antagonismes assure l'éveil d'une conscience neuve et permet le développement de la démocratie. Mais comment unifier une société si la lutte pour le pouvoir est une affaire d'ethnie, comment développer des solidarités si le combat politique se transforme en une arène où n'entrent que des gladiateurs ?A dire vrai , quand les contestations et les antagonismes se règlent les armes à la main, dans le sang en dehors de toute éthique, on est hors de la politique. La politique moderne est civilisée et se doit de substituer la discussion aux fusils, le dialogue aux rebellions ;elle est effort sans fin pour éliminer la violence, du moins pour la restreindre. Son rôle est de modérer les conflits par des compromis en vue de favoriser une intégration dans laquelle chacun des membres de la société se sent comme acteur de la vie politique, où chacun trouve un épanouissement total de son être dans une sorte d'interaction circulaire sans bornes. «La vie en commun, déclarait Julien FREUND, n'est pas possible sans arrangement à l'amiable, sans conventions, sans accomodements parfois confus ou sans concessions réciproques $» .^{17}$

24 Si les conflits sont récurrents en Afrique, cela est assurément dû aux problèmes politiques mal résolus. Ceux-ci exigent que l'on fasse voir les choses essentiellement sous l'angle de l'intégration et de la concorde. Il est temps de se poser toujours la question fondamentale suivante :quel est le but de la politique en Afrique ?Ce but devrait se résumer en deux points essentiel, pour qui veut adhérer à la conception hobbienne du contrat : la sécurité et la prospérité. La sécurité d'abord, parce qu'il n'y a pas de développement harmonieux, si le peuple ne se sent pas protégé contre des agressions extérieures et intérieures; si le maintien de l'ordre n'est pas assuré convenablement, il n'y a pas de paix. La sécurité implique le respect des lois et la lutte 
contre l'impunité afin de valoriser la liberté d'aller et de venir, la liberté d'expression, etc. Sans sécurité, il ne saurait avoir de concorde véritable dans la mesure où la concorde suppose qu'on ait au préalable surmonté les inimitiés internes. La concorde n'est- elle pas «un vivre ensemble», un bien commun, une communauté de vie et d'amitié ?Par son action de vaincre l'inimitié et d'établir la paix, la sécurité attirera à coups sûrs la prospérité car une collectivité a besoin de bonne conscience pour s'épanouir et obéir à l'autorité. On pourrait donc dire avec Julien FREUND que le but spécifique du politique «consiste dans la volonté d'une unité politique de conserver son intégrité et son indépendance dans la concorde intérieure et la sécurité extérieure ${ }^{18}$.

25 Mais si la population ne gagne pas sa vie mais perd sa liberté, si elle n'est pas dans une loge sécurisante, elle met en jeu sa vie pour assurer sa survie. Cette triste condition entraîne la non-reconnaissance du pouvoir et le pourrissement de l'État. Le rapport de force s'articule en concurrence et les oppositions se détruisent pour n'apparaître que sous les flammes de la contention et du conflit. Le consentement devient une chimère et la légitimité une fiction stérile. Dans un tel cas, le pouvoir n'est plus en mesure de faire respecter la loi et l'Ėtat perd toute prérogative dans la mesure où le droit du " soulèvement » devient légitime. Il naît alors l'équilibre de la terreur où tout suspect devient coupable où les joies paisibles se transforment en océan de larmes et la perpétuation de l'horreur. L'État devient son propre glaive parce que la démesure s'est retournée contre elle-même.

26 Après le Rwanda, le Burundi, la Sierra Leone et le Liberia, l'échec du langage et de la culture n'est plus à démontrer en Afrique. Il y a comme une sorte de stagnation dans les cercles de l'enfer. Ainsi, l'autoréflexion du penser politique doit aussi penser contre soi-même pour analyser l'expérience de la Séparation, pour comprendre l'imprudence du langage et ses détours rusés. Les interprétations et les modifications tendancieuses des textes constitutionnels, les ambiguïtés des Accords, le non respect des engagements et de la parole donnée constituent les fondements de la déréliction politique dont la fin est les guerres civiles, justement parce que les lois sont devenues folles et les légitimités enterrées dans des purgatoires. «Ce travail de débordement qu'est la ruse, la raison le constate à travers ses méprises et les refoulements d'un discours qui n'aboutit qu'à travers les trajectoires brisées qui ne peuvent jamais se saisir dans le simple déploiement des sens. Lectures plurielles, ambiguïté du sens immédiat, multiplicité des points de vue accompagnent tout essai d'interprétation ${ }^{19}$.

Dans notre impuissance à étouffer notre vacarme langagier, nous nous sommes laissés emporter par les hésitations stériles d'une conscience déchirée, haineuse, délictueuse. Le rejet de certaines valeurs normatives nous a conduit à toucher au fruit défendu, à entrer petitement dans le cercle fiévreux des crises politiques dont les issues sont connues :rebellions, génocide, coups d'Ėtats, guerres civiles. Le piétinement de la morale par notre conscience malheureuse a entériné l'assassinat des droits, même les plus élémentaires, le "droit »de vivre, d'exister et même de porter son nom, le « droit » de nommer où d'appartenir à une ethnie. Cette déréliction est-elle excusable ? C'est ici que nous rattrape notre faute vénielle: « Le Triomphe de l'Identitaire ${ }^{20}$, selon l'expression de Henri LEFEBVRE. Le Nouveau Testament exprimera une idée analogue en utilisant le terme de PROSOPOLEPSIE. «La prosopolepsie, selon Vladimir JANKÉLÉVITCH, est la duperie qui consiste à faire acception du masque (...), à prendre en considération le faciès et la couleur de la peau, autrement dit le personnage. 
Prosopon est en somme une apparence superficielle. Ce qui est inessentiel et accidentel, ce qui est grimace ou appartenance « adjectivale »,Dieu n'en tient pas compte :Dieu ne tient compte que de l'essence, il ne tient compte que de l'humanité de l'homme, sans considérer la pigmentation de sa peau ni la forme de son nez $»^{21}$.

Conclusion

28 L'Afrique semble aller à vau l'eau en se perpétuant dans les crises politiques infinies. Cet état critique et les tensions qui s'entremêlent font désespérer d'un continent qui est en train de perdre son ancienne base de grandeur morale pour s'enraciner dans les fondements de ressentiment, de domination et de volonté de puissance. Or, ces fondements montrent leur fragilité et leur impuissance lorsque des crises subsistent. La gestion de ces crises aboutit à un terrorisme particulier, elle entretient la psychose d'une guerre civile aux contours indéterminés. Cette psychose atteint son pourrissement par l'auto-exclusion de certaines masses de la population qui craignent l'extermination et se résignent à la forfaiture du cercle de fer. Cela dénote de l'inintelligibilité rationnelle et de la méprise de la société civile par les hommes politiques. Jusqu'alors, le langage s'impliquait dans la pratique politique en Afrique sous l'arbre à palabres pour résoudre les différends. Mais il semble, aujourd'hui, que ce langage s'est détaché pour n'apparaître que comme un venin, pour devenir une déréliction. La crise des référentiels a entraîné le Déchirement du LOGOS. «Très lentement, on s'aperçut que le langage - le discours - reste en l'air, seul, sans règle autre qu'interne ( la logique), sans certitude autre que sa confiance en lui, sauf si l'on admet l'équivalent des équivalents :l 'Etat. Très lentement, on s'aperçut que la priorité simultanée du discours et de la science du discours (la logologie) accompagnait le meurtre du langage et l'acceptation du conventionnel (le n'importe - quoi - isme !). A chacun son langage et son discours pour dire n'importe quoi $»^{22}$. Que faire ? Il faut repenser le penser politique en Afrique en apprenant à parler aux Autres. Le moment est enfin arrivé pour s'occuper des «Laissés-Pour-Compte » et des exclus de la société, pour promouvoir la culture de «l'homme de qualité », très sainement.

\section{BIBLIOGRAPHIE}

Arendt (H.).- Le système totalitaire , Traduit de l'américain par Jean- Loup Bourger , Robert Davreu \& Patrick Lévy (Paris, Seuil,1972), 315 pages.

Brun (J.).- Les conquêtes du monde et la séparation ontologique (Paris, PUF, 1961 ), 298 pages.

Deleuze (G.)\&Guattari (P.).- Qu'est-ce que la philosophie ? (Paris, Minuit, 1991), 207 pages.

Duverger (M.).- Introduction à la politique (Paris, Gallimard, 1964), 382 pages.

Fontaine (Jean de la).-'Le corbeau et le renard" in Les fables (Paris, Garnier -Flammarion , 1966), 382 pages.

Freund (J.).- Qu'est-ce que la politique? (Paris, Sirey, 1965), 189 pages.

Jankélévitch(V.).- Le paradoxe de la morale (Paris, Seuil, 1981), 191 pages. 
Lefebvre (H.).- De l'Etat 4.Les contradictions de l'Etat moderne (Paris, UGE , 1978,Coll. 10/18), 467 pages.

Marcuse (H.).- contre - révolution et révolte, traduit de l'anglais par Didier Coste (Paris, Seuil,1972), 167 pages.

Rey(J.M).- L'enjeu des signes_(Paris, Seuil,1971), 285 pages.

Schroyer (T.).- Critique de la domination, Traduit de l'américain par Jacques Debousy, (Paris, Payot, 1980), 267 pages.

Védrine (H.).- Les ruses de la raison (Paris, pbp, 1982), 243 pages.

\section{NOTES}

1. Duverger (M.).-Introduction à la politique (Paris, Gallimard, 1964),pp.163-164

2. Brun (J.).-Les conquêtes du monde et la séparation ontologique (Paris, PUF, 1961 ), p. 238

3. Brun, op.cit.,p.234.

4. Marcuse (H.) .-contre-révolution et révolte, traduit de l'anglais par Didier Coste (Paris, Seuil,1972), pp.73-74.

5. Sur les campus universitaires de la Côte d'Ivoire, en 1999-2000, les machettes s'étaient substituées aux arguments pour contraindre soit des dissidents de la Fédération Estudiantine et Scolaire de Côte d'Ivoire(FESCI) qu'on suspecte d'être des bras séculiers de l'opposition radicale, à demeurer des acteurs passifs au sein du syndicat estudiantin, soit pour se démarquer des autres qu'on soupçonne d'être des « taupes » du régime au pouvoir, un groupe de pression contre-révolutionnaire pour dissuader toute velléité de l'opposition. Cette perversion du penser, entérinée par la déréliction du langage, est la copie conforme du déséquilibre des intellectuels dans une société multi-ethnique où il ne suffit qu'une étincelle pour allumer la paille. Cette déréliction du langage dans la perversion du penser politique a eu pour conséquence la déréliction politique dont l'une des figures les plus hideuses est la rébellion armée du 19septembre 2002 dans laquelle l'on retrouve les protagonistes de la crise estudiantine dans un camp comme dans l'autre.

6. Deleuze (G.)\&Guattari (P.).-Qu'est-ce que la philosophie ? (Paris, Minuit, 1991), p.191.

7. Fontaine ( Jean de la).-Le corbeau et le renard in Les fables (Paris,Garnier-

Flammarion,1966), p.52.

8. Jankélévitch(V.).-Le paradoxe de la morale (Paris, Seuil, 1981), p.32.

9. Duverger (M.), op.cit.,pp.251-252.

10. Marcuse(H.), op.cit., p.25.

11. Arendt(H.).-Le système totalitaire , Traduit de l'américain par Jean-Loup Bourger , Robert Davreu\& Patrick Lévy(Paris, Seuil,1972), p.205

12. Schroyer (T.).-critique de la domination, Traduit de l'américain par Jacques

Debousy, (Paris,Payot, 1980), p.154.

13. Rey(J.M).-L'enjeu des signes(Paris, Seuil,1971), p. 18

14. Op.cit., p.12

15. Arendt (H.), op.cit., p.116

16. Marcuse(H.), op.cit., p.68

17. Freund (J.)-Qu'est-ce que la politique? (Paris, Sirey, 1965), p.78.

18. Op.cit., p.34. 
19. Védrine (H.).-Les ruses de la raison (Paris, pbp, 1982),p.70.

20. Lefebvre (H.).-De l'Etat 4.Les contradictions de l'Etat moderne (Paris, UGE , 1978,Coll. 10/18), p.224.

21. Jankélévitch (V.), op.cit.,p.42.

22. Lefebvre (H.) , op.cit. ; p.219.

\section{RÉSUMÉS}

Le continent africain est en butte à de nombreuses crises politiques. Ces crises ont pour fondement la déréliction du langage. Il faudrait donc repenser le dire politique afin d'instaurer la concorde et la sécurité entre les peuples, gages de tout développement harmonieux et de paix durable. Le but du politique est de faire en sorte que la démocratie amenuise les tensions au lieu de les exacerber. Notre sens de l'honneur exige de nous la moralisation de notre penser politique pour re-construire « le - vivre - ensemble » en toute liberté.

\section{AUTEUR}

\section{DR SAMBA DIAKITE}

Enseignant-Chercheur Université de Bouaké Côte d'Ivoire docteursamb@yahoo.fr 